\title{
Effect of First Line Gastric Cancer Chemotherapy Regime on the AGS Cell Line - MTT Assay Results
}

\author{
Reza Alizadeh-Navaei ${ }^{1}$, Alireza Rafiei ${ }^{1 *}$, Saeid Abedian-Kenari ${ }^{2}$, Hossein \\ Asgarian-Omran ${ }^{1}$, Reza Valadan ${ }^{1}$, Akbar Hedayatizadeh-Omran ${ }^{1}$
}

\begin{abstract}
Background: Combination chemotherapy regimes are common treatments for cancer. The aim of this study was to evaluation the effect of individual chemotherapeutic agents in comparison with a first line chemotherapy regime treatment in the AGS gastric cancer cell line by MTT assay. Materials and Methods: In this experimental study, AGS cells were grown in RPMI-1640 supplemented with $10 \%$ fetal calf serum and $100 \mathrm{IU} / \mathrm{ml}$ penicillin, and $10 \mu \mathrm{g} / \mathrm{ml}$ streptomycinin, under a humidified condition at $37^{\circ} \mathrm{C}$ with $5 \% \mathrm{CO}$. All cells were washed with PBS and detached with trypsin, centrifuged and 8000 cells re-plated on to 96- well plates. LD50 doses of Epirubicin, Cisplatin and 5-fluorouracil were added to each well in mono or triple therapy. Anti-proliferative activities were determined by MTT assay after 24, 48 or $72 \mathrm{~h}$. Results: Results of MTT assays showed that there were no significant differences among 3 drugs in monotherapy $(p=0.088)$, but there was significant difference between combination therapy with epirubicin $(P=0.031)$ and $5 F U(p=0.013)$ on cell survival at $24 \mathrm{~h}$. After 48 and 72 hours, cell viability showed significant differences between the 3 drugs $(p=0.048$ and $P=0.000$ for 48 and $72 \mathrm{~h}$, respectively) and there was significant difference between combination therapy with epirubicin $(P=0.035$ and $P=0.002$ for 48 and $72 \mathrm{~h}$, respectively). Conclusions: The results showed no significant differences between these chemotherapy drugs each given alone, but combination therapy with 3 drugs had significant effects on cell viability in comparison with epirubicin alone.
\end{abstract}

Keywords: Gastric cancer - chemotherapy - AGS cell line - MTT - monotherapy - combined therapy

Asian Pac J Cancer Prev, 17 (1), 131-133

\section{Introduction}

Gastric cancer is the third most common cause of death worldwide, that affecting both people with low socioeconomic status and developing countries (Arnold et al., 2014; Guedes et al., 2014). Gastric cancer is accounting for $8 \%$ of cancer incidence and $10 \%$ of cancer deaths (Lin et al., 2015). Several risk factors for gastric cancer have been identified, including Helicobacter pylori infection, salt-preserved foods, dietary nitrite, smoking, alcohol, obesity, radiation, and family history (Tsugane et al., 2004). The incidence is particularly high in East Asia, Eastern Europe, and parts of Central and South America, and it is about twice as high among men than among women. Prognosis is generally rather poor, with 5 -year relative survival below $30 \%$ in most countries (Brenner et al., 2009). Researchers also found that the incidence rates of gastric cancer varied across different geographic regions and this variation may be associated with genetic, lifestyle or environmental factors, including diet (Armstrong and Doll, 1975). Treatment strategy in gastric cancer includes surgery, radiation therapy, chemotherapy, or targeted therapy (Choi et al., 2015). Chemotherapy remains the cornerstone of treatment for gastric cancer patients with locally advanced and metastatic disease. Many chemotherapy agents have activity including platinums, irinotecan, fluorouracil, taxanes and anthracyclines. Treatment with a combination of three agents has been shown to lead to modest improvements in survival compared to two agents, but at the expense of significant toxicity (Ajani et al., 2007). Combination chemotherapy may improve the length and quality of survival of some patients with advanced gastric cancer. First line chemotherapy regimens for gastric cancer include epirubicin, cisplatin and 5-fluorouracil (Kim, 2005; Benson, 2008).

There was some combination chemotherapy in chemotherapy regime treatment, so the aim of this study was to evaluation the effect of each individual chemotherapeutic agent in LD50 in comparisons with first line chemotherapy regime treatment in the AGS gastric cancer cell line by MTT assay.

\section{Materials and Methods}

In this experimental study, AGS cells (NCBI No.C131, Human gastric adenocarcinoma cell line) was purchased from Pasteur Institute of Iran (Pasteur Institute, Tehran, 


\section{Reza Alizadeh-Navaei et al}

Iran). The cells were grown in RPMI-1640 supplemented with $10 \%$ fetal calf serum and $100 \mathrm{IU} / \mathrm{ml}$ penicillin, and $10 \mu \mathrm{g} / \mathrm{ml}$ streptomycin (PAA, Pasching, Austria) in flask(Nunc,Denmark). The cells were cultured in a humidified condition at $37^{\circ} \mathrm{C}$, and $5 \% \mathrm{CO}_{2}$. When the cultures were $80-90 \%$ confluent, All cells were washed with PBS $(\mathrm{pH}=7.4)$, detached with $0.25 \%$ trypsin, centrifuged at1200 rpm for $5 \mathrm{~min}$ and re-plated onto 96- well plates and returned to the incubator for $24 \mathrm{~h}$ to allow celladherence to the plate. Cell counts were performed using a haemocytometer and 8000 cells were used for all experiments.Then, LD50 doses of three chemotherapeutic agents including Epirubicin, Cisplatin and 5-fluorouracilwere added to each well in mono or triple therapy and the plate returned to the incubator for either 24,48 or $72 \mathrm{~h}$. Anti-proliferative activities of mono or triple therapy on the AGS cell line, was determined by 3-4, 5-dimethylthiazol-2-yl 2,5-iphenyltetrazolium bromide (MTT) colorimetric assay. After 24, 48 or $72 \mathrm{~h}$ of exposure to the chemotherapy agents, MTT (Sigma) was added and then incubated for a further $4 \mathrm{~h}$ at $37 \mathrm{C}$. After wards, $20 \mu \mathrm{l}$ of MTT solution $(5 \mathrm{mg} / \mathrm{ml}$ in PBS) was added to each well and incubated for an additional 4 $\mathrm{h}$ followed by adding $200 \mu \mathrm{l}$ of MTT solvent and shacked for 10 minutes. Then, spectrophotometric absorbance was measured at $570 \mathrm{~nm}$ andread by ELISA reader and Optical density (OD) for each well was determined.

There was a control sample which remained untreated and received the equal volume of medium. All different treatment carried out in triplicate.

CellViability was determined using the following formula:

Cell Viability $(\%)$ in compares to the controls $=\frac{\text { OD of cell in each well }}{\text { Mean OD of control cells }} * 100$

Data were analyzed by SPSS software (version 15). All results were expressed as mean $\pm \mathrm{SD}$. The significance of difference was evaluated with ANOVA and Tukey test. Significant difference was set at $\mathrm{P}<0.05$.

\section{Results}

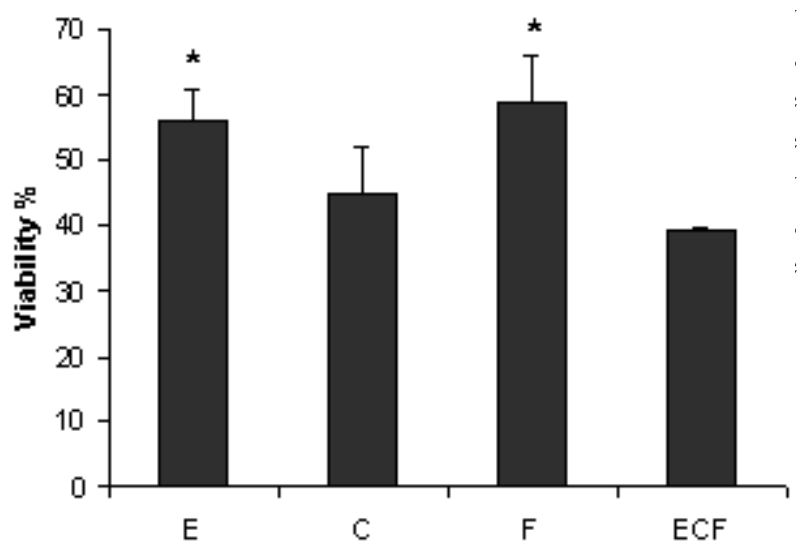

Figure 1. The effect of chemotherapy drug on viability of AGS by MTT assay at $24 \mathrm{~h}$. * Significant difference between combination therapy with epirubcin $(\mathrm{P}=0.031)$ and $5 \mathrm{FU}$ $(\mathrm{p}=0.013)$. E: Epirubicin, C: Cisplatin, F: 5-fluorouracil, ECF: Epirubicin + Cisplatin + 5-fluorouracil

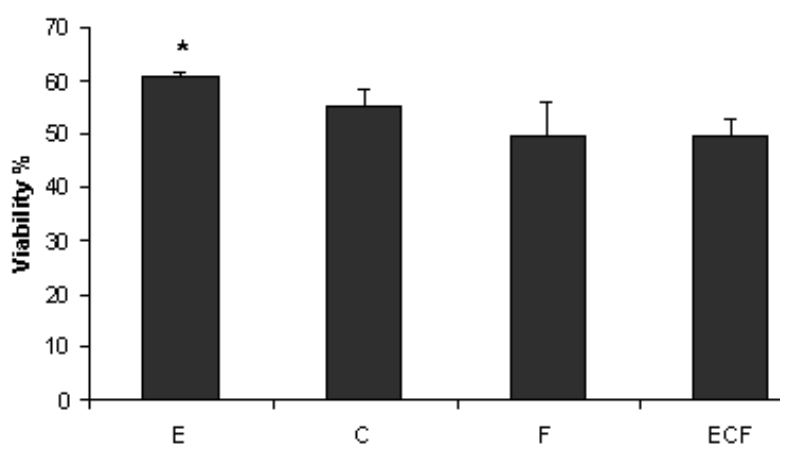

Figure 2. The effect of chemotherapy drug on viability of AGS by MTT assay at $48 \mathrm{~h}$. * Significant difference between combination therapy with epirubcin $(\mathrm{P}=0.035)$. E: Epirubicin, C: Cisplatin, F: 5-fluorouracil, ECF: Epirubicin + Cisplatin + 5-fluorouracil

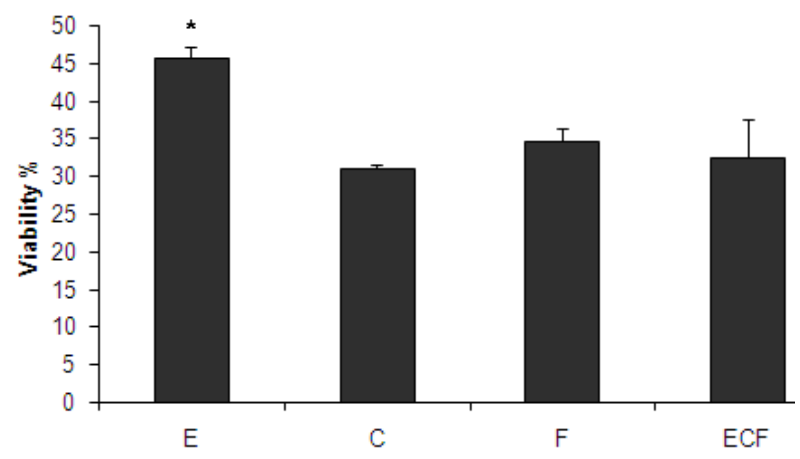

Figure 3. The Effect of Chemotherapy Drug on Viability of AGS by MTT assay at 72 h. * Significant difference between combination therapy with epirubcin $(\mathrm{P}=0.035) \mathbf{E}$ : Epirubicin, C: Cisplatin, F: 5-fluorouracil, ECF: Epirubicin + Cisplatin +5 -fluorouracil

of living AGS cells compared to control showed significant difference between 3 drugs at concentrations of LD50 of each chemotherapy drugs $(\mathrm{p}=0.048)$. In this time, there was significant difference between combination therapy with epirubcin $(\mathrm{P}=0.035)$. Cell viability changes in third day were shown in figure 3. Percentage of surviving AGS and cells showed significant difference between 3 drugs $(\mathrm{p}=0.000)$. Also, There was significant difference between combination therapy with epirubcin $(\mathrm{P}=0.002)$

\section{Discussion}

Chemotherapy for gastric cancer is varied, with most regimes including one of epirubicin, cisplatin or 5-FU (Lordick and Siewert, 2006) and the aim of this study was to evaluation the effect of each individual chemotherapeutic agent in LD50 in comparisons with first line chemotherapy regime treatment in the AGS gastric cancer cell line by MTT assay. Result of present study show that, after 48 $\mathrm{h}$ the cell viability was difference between epirubicin, cisplatin and 5-fluorouracil in monothearpy and cell viability was lowest in cisplatin group. In comparison between monotherapy and combinational regime, there was significant difference between combination therapy with epirubcin and cell viability combination therapy 
and monotherapy by cisplatin or 5-fluorouracil was not significant difference in 48 and $72 \mathrm{~h}$, especially. Similar to uor result, in Weinreich et al. study reported that replacing 5 -FU in ECF (epirubicin + cisplatin + 5-fluorouracil ) with a novel duplex drug resulted in greater growth inhibition of cancer cells than of the non-malignant cell lines and the inversion of the chemosensitivity of MKN45 and 23132/87 cells (Weinreich et al., 2015). But, in Suttie et al study, among three these chemotherapeutic agents, epirubicin having the greatest effectin AGS cells, followed by 5-fluorouracil then cisplatin (Suttie et al., 2007). Previous studies show that exposure to epirubicin and cisplatin induced $\mathrm{G} 1$ and G2 cell cycle arrest (Zoli et al., 2004; Suttie et al., 2007). Epirubicin, an anthracycline derivative of doxorubicin effects as a DNA intercalating agent and as an inhibitor of topoisomerase II (Zoli et al., 2004). Cisplatin as an alkylating agent binds to DNA within G2, forming cisplatin-DNA adducts which causes an alteration in the conformation of DNA leading to cell cycle arrest and apoptosis (Suttie et al., 2007). It is reported that action of 5-FU is related to its conversion to 5-fluoro2'-deoxy-5'-monophosphate leading to the inhibition of thymidylate synthase and hence DNA synthesis (Pinedo and Peters, 1988). Studies have described an increase S-phase fraction in tumour cells, caused by 5-fluorouracil (Yamane et al., 1999; Park et al., 2004).

One of the limitations of our study is that we didn't use normal cell line for comparison but we have negative control as control group for chemotherapy drug on wellperfused cells. In conclusion result of present study show that there was no significant difference between these chemotherapy drugs, but combination therapy with 3 drugs had significant effect on cell viability in comparison with epirubicin.

\section{Acknowledgements}

This study has been extracted from the $\mathrm{PhD}$ thesis of Reza Alizadeh-Navaei and was supported by Grant Number 92-64 from Mazandaran University of Medical Sciences, Iran.

\section{References}

Ajani JA, Moiseyenko VM, Tjulandin S, et al (2007). Clinical benefit with docetaxel plus fluorouracil and cisplatin compared with cisplatin and fluorouracil in a phase III trial of advanced gastric or gastroesophageal cancer adenocarcinoma: the V-325 Study Group. J Clin Oncol, 25, 3205-9.

Armstrong B, Doll R (1975). Environmental factors and cancer incidence and mortality in different countries, with special reference to dietary practices. Int J Cancer, 15, 617-31.

Arnold M, Moore SP, Hassler S, et al (2014). The burden of stomach cancer in indigenous populations: a systematic review and global assessment. Gut, 63, 64-71.

Benson AB (2008). Advanced gastric cancer: an update and future directions. Gastrointest Cancer Res, 2, 47-53.

Brenner H, Rothenbacher D, Arndt V (2009). Epidemiology of stomach cancer. Methods Mol Biol, 472, 467-77.

Choi YY, Noh SH, Cheong JH (2015). Evolution of Gastric Cancer Treatment: From the Golden Age of Surgery to an
Era of Precision Medicine. Yonsei Med J, 56, 1177-85.

Guedes MT, de Jesus JP, de Souza Filho O, et al (2014). Clinical and epidemiological profile of cases of deaths from stomach cancer in the National Cancer Institute, Brazil. Ecancermedicalscience, $8,445$.

Kim YH (2005). Chemotherapy for advanced gastric cancer: slow but further progress. Cancer Res Treat, 37, 79-86.

Lin X, Zhao Y, Song WM, et al (2015). Molecular classification and prediction in gastric cancer. Comput Struct Biotechnol $J, \mathbf{1 3}, 448-58$.

Lordick F, Siewert JR (2006). [Perioperative chemotherapy vs. surgery alone in resectable gastroesophageal carcinomas. Results of the MAGIC study]. Chirurg, 77, 1166-7.

Park JK, Lee SH, Kang JH, et al (2004). Synergistic interaction between gefitinib (Iressa, ZD1839) and paclitaxel against human gastric carcinoma cells. Anticancer Drugs, 15, 809-18.

Pinedo HM, Peters GF (1988). Fluorouracil: biochemistry and pharmacology. J Clin Oncol, 6, 1653-64.

Suttie SA, Park KG, Smith TA (2007). [18F]2-fluoro-2-deoxyD-glucose incorporation by AGS gastric adenocarcinoma cells in vitro during response to epirubicin, cisplatin and 5-fluorouracil. Br J Cancer, 97, 902-9.

Tsugane S, Sasazuki S, Kobayashi M, et al (2004). Salt and salted food intake and subsequent risk of gastric cancer among middle-aged Japanese men and women. Br J Cancer, 90, 128-34.

Weinreich J, Archid R, Bajaeifer K, et al (2015). Growth and Chemosensitivity of Gastric Adenocarcinoma and NonMalignant Cell Lines in Response to Novel Anti-Cancer Drug Combinations. Chemotherapy, 60, 346-52.

Yamane N, Makino M, Kaibara N (1999). S-phase accumulation precedes apoptosis induced by preoperative treatment with 5 -fluorouracil in human colorectal carcinoma cells. Cancer, 85, 309-17.

Zoli W, Ricotti L, Tesei A, et al (2004). Schedule-dependent cytotoxic interaction between epidoxorubicin and gemcitabine in human bladder cancer cells in vitro. Clin Cancer Res, 10, 1500-7. 